\title{
Release from short-term proactive interference with change in item duration
}

\author{
J. J. CREMINS \\ Boston College, Chestnut Hill, Massachusetts 02167 \\ and \\ M. T. TURVEY \\ University of Connecticut, Storrs, Connecticut 06268 \\ and Haskins Laboratories, New Haven, Connecticut 06511
}

\begin{abstract}
The results of three experiments suggest that in the release from proactive interference paradigm, increasing item duration facilitates recall but decreasing item duration does not. Two different interpretations of these findings were suggested: an explanation in terms of modification of encoding strategy and an explanation in terms of novelty-arousal.
\end{abstract}

The release from proactive inhibition (PI) paradigm permits the examination of the hypothesis that the memory trace is composed of a conglomerate of features or attributes. Studies using this procedure have delineated a number of coding dimensions in short-term memory for verbal items (Wickens, 1970, 1972, 1973). One generalization that emerges when comparing the amount of release obtained with different feature shifts is that changes in syntactic features yield little or no release, changes in semantic features yield substantial amounts of release, and changes in features associated with the physical characteristics of the presentation event yield intermediate amounts of release (Gardiner, Ulee, Redman, \& Ball, 1976). Therefore, it appears that verbal items in short-term memory (STM) may be encoded via meaning, parts of speech, or even modality.

One question that has not previously been asked, however, is one regarding time; precisely, is item duration a coding dimension in STM?

\section{EXPERIMENT 1}

The first study was an attempt to assess the possibility that increasing the duration of item presentation could induce PI release.

\section{Method}

The subjects were 60 students, male and female, randomly selected from the introductory psychology classes at the University of Connecticut. Each subject received five STM tests. An STM test was composed of the following events presented as a sequence of slides and delivered to the subject by means of a Kodak Carousel projector controlled by a tape timer: First, an asterisk of $2 \mathrm{sec}$ duration acted as a ready signal. Second, a consonant trigram of a specified duration was read aloud once by the subject. In the experimental condition, the duration of this event was $1.5 \mathrm{sec}$ for STM Tests $1-4$ and $2.5 \mathrm{sec}$ for STM

The experiments reported in this paper were completed while the senior author was a student at the University of Connecticut.
Test 5, while for the control group, it was $2.5 \mathrm{sec}$ for all five tests. Third, a three-digit number was present through the retention interval of $8 \mathrm{sec}$. The subject read this number aloud and counted back aloud from this number by threes. Fourth, a question mark cued the subject to recall the consonant trigram. The duration of the recall phase was $10 \mathrm{sec}$. There was no intertest interval as such. On termination of the recall phase, the ready signal for the next STM test appeared. The subject's recall of the trigram and performance on the interpolated task were recorded by the experimenter.

The same five trigrams were employed in both the experimental and control conditions and were counterbalanced across the five STM tests. A different three-digit number appeared on each test. Subjects were allocated by order of appearance at the laboratory to one of the two conditions with 30 subjects per condition. Subjects in both conditions received the same set of instructions prior to the beginning of the experiment. At completion of STM Test 5, subjects in both conditions were asked if they detected a difference between the presentation of the consonant trigram on STM Test 5 and the presentation of the previous trigrams.

\section{Results}

Each subject's recall on each STM test was scored in the manner used by Wright (1967). A value of two was assigned to a correct item recalled in its correct trigram position; a value of one was assigned to an item correct for that trigram, but recalled in an incorrect trigram position; and a value of zero was assigned to an incorrect item or an omission. Figure 1 represents the data for STM Tests 14 and the critical test, Test 5.

The data of STM Tests 1.4 were subjected to an analysis of variance. No significant difference was obtained between the experimental and control conditions $[F(1,58)=3.05, p>.05]$. The effect of tests was significant $[\mathrm{F}(3,174)=95.84, \mathrm{p}<.001]$, demonstrating that PI had occurred, but the condition by STM tests. effect was insignificant. Therefore, the fact that the experimental and control groups differed with respect to the presentation duration did not produce a difference in the retention over trials function. 


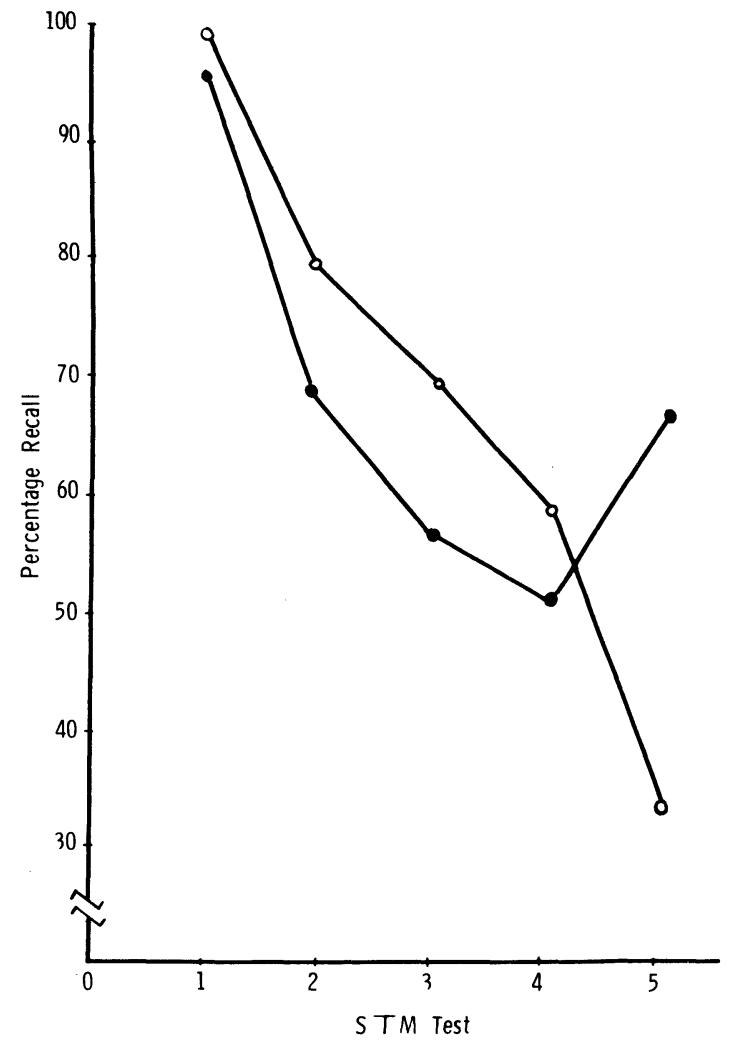

Figure 1. Recall as a function of STM test in Experiment 1. Cosed circles represent the experimental group (shift from 1.5 to $2.5 \mathrm{sec}$ ); open circles represent the control group ( $2.5 \mathrm{sec}$ throughout).

The difference between the recall scores on STM Test 5 for the experimental and control conditions was analyzed by means of a t test. This difference was found to be highly significant $[\mathrm{t}(29)=15.70, \mathrm{p}<.001]$. However, despite the superior performance on Trial 5 , not one of the subjects in the experimental condition detected any difference between the presentation on Test 5 and the presentations on Tests 14 . To insure that the difference in recall on Test 5 was not due to differences in rehearsal, a t test was performed on the interpolated activity scores for the experimental and control condition on this trial. No significant difference was obtained. Finally, we may note that while the release was significant, it was not exceptional. It is comparable to the intermediate amounts of release found with a shift in physical characteristics (see Gardiner et al., 1976; Wickens, 1972).

\section{EXPERIMENT 2}

Experiment 1 showed that a transition from a history of items of $1.5 \mathrm{sec}$ duration to an item of $2.5 \mathrm{sec}$ duration produced the PI release phenomenon. If shift in duration acts in a manner analogous to shift in class of material (Wickens, 1970, 1972, 1973), then a transition from a history of items of $2.5 \mathrm{sec}$ duration to an item of $1.5 \mathrm{sec}$ duration should produce the same release from PI. A second experiment was conducted to assess this prediction.

\section{Method}

The apparatus, materials, and procedure were the same as for Experiment 1. However, for the second experiment, the duration of trigrams on STM Tests 1-4 of the experimental condition was $2.5 \mathrm{sec}$, with $1.5 \mathrm{sec}$ duration for the trigram on STM Test 5 . In the control condition, the trigram duration was held constant at $1.5 \mathrm{sec}$ for all five STM tests.

Fifty subjects were randomly selected from the same subject pool used in Experiment 1, with 25 subjects allocated to each condition.

\section{Results}

The recall data were scored in the same manner as that employed in Experiment 1. An analysis of variance on STM Tests 1.4 revealed that only the tests effect was significant $(\mathrm{p}<.001)$, thus corroborating the data of Experiment 1. Figure 2 represents the recall data for Tests $1-4$ and Test 5 . Figure 2 clearly indicates that no release from PI was obtained on Test 5.

\section{EXPERIMENT 3}

A third experiment was conducted that included both conditions of increasing and decreasing item duration. These conditions had been examined separately in Experiments 1 and 2.

\section{Method}

The subjects were 120 students, male and female, randomly selected from the introductory psychology classes at the Uni-

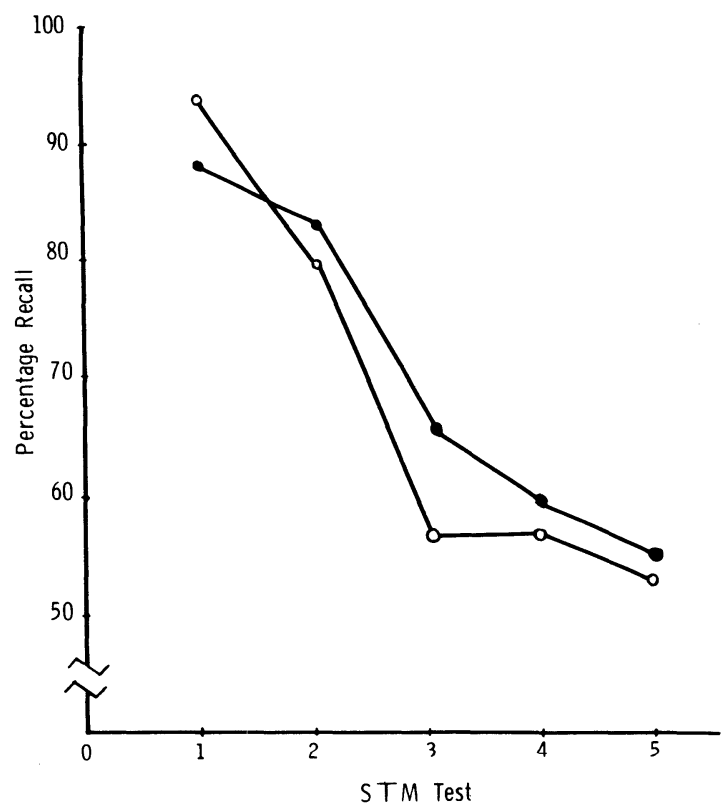

Figure 2. Recall as a function of STM test in Experiment 2. Closed circles represent the experimental group (shift from 2.5 to $1.5 \mathrm{sec})$; open circles represent the control group (1.5 sec throughout). 


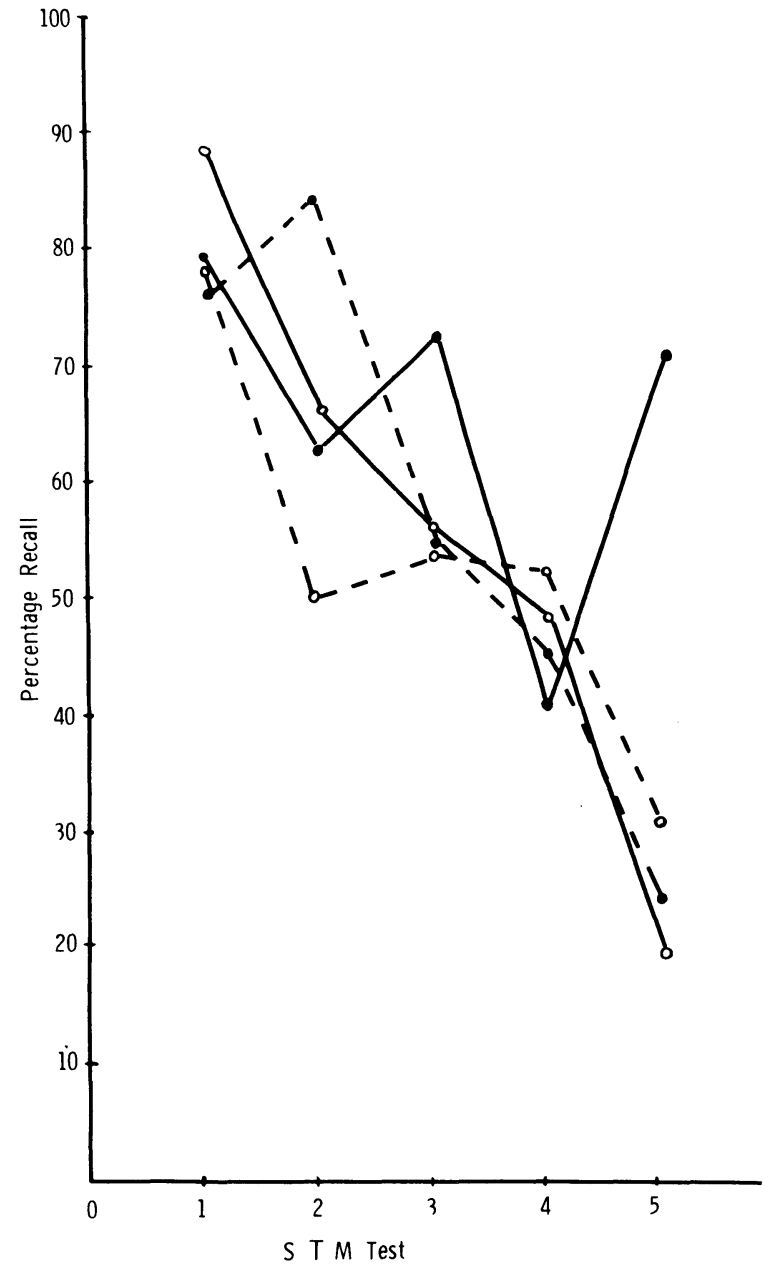

Figure 3. Recall as a function of STM test in Experiment 3. Cosed circles with solid lines represent a shift from $1.5 \mathrm{sec}$ to $2.5 \mathrm{sec}$; open circles with solid lines represent a control condition of 2.5 sec throughout. Closed circles with broken lines represent a shift from 2.5 to $1.5 \mathrm{sec}$; open circles with broken lines represent a control condition of $1.5 \mathrm{sec}$ throughout.

versity of Connecticut. Each subject received five STM tests. A test was composed of the following events presented as a sequence of slides and delivered to the subject by means of a Kodak Carousel projector controlled by a tape timer: First, an asterisk of $2 \mathrm{sec}$ duration acted as a ready signal. Second, a consonant trigram of a specified duration was read aloud once by the subject. In the experimental condition, the duration of each trigram was $1.5 \mathrm{sec}$ for Tests $1-4$ and $2.5 \mathrm{sec}$ for Test 5 or $2.5 \mathrm{sec}$ for Tests $1-4$ and $1.5 \mathrm{sec}$ for Test 5 . In the control conditions, the duration of each trigram was either $2.5 \mathrm{sec}$ or $1.5 \mathrm{sec}$ for all five tests. Third, a series of eight two-digit numbers was presented at $1-\mathrm{sec}$ intervals during the 8 -sec retention interval. Subjects were required to sum and classify each number as odd or even. Fourth, a question mark cued the subject to recall the consonant trigram. The duration of the recall phase was $10 \mathrm{sec}$. There was no intertest interval as such. On termination of the recall phase, the ready signal for the next trial appeared. The subject's recall of the trigram and performance on the interpolated task were recorded by the experimenter.

The same five trigrams were employed in both the experimental and control conditions and were counterbalanced across the five tests. A different set of two-digit numbers appeared on each trial. Subjects were allocated by order of appearance at the laboratory to one of the four conditions, with 30 subjects per condition. Subjects in each condition received the same set of instructions prior to the beginning of the experiment. At the completion of Test 5 , subjects in both experimental conditions were asked if they detected a difference between the presentation of the consonant trigram on Test 5 and the presentation of the previous trigrams.

\section{Results}

Recall was scored by the method described previously; the data are given in Figure 3. The recall data for Tests $1-4$ were subjected to an analysis of variance. No significant difference was obtained between the experimental and control conditions, $[\mathrm{F}(3,119)=2.8842$, $p>.05]$. The tests effect was significant $[F(4,64)=$ 26.3352, $\mathrm{p}<.001$ ], demonstrating that PI had occurred.

The Test 5 recall data were analyzed by means of $\mathrm{t}$ tests. This analysis revealed that increasing item duration produced PI release $[\mathrm{t}(59)=5.47, \mathrm{p}<.001]$, while decreasing item duration had no effect on recall.

Subjects did not report awareness of the changes in duration.

\section{DISCUSSION}

The results of these studies demonstrate that PI release occurs when duration of item presentation is increased but does not occur when duration is decreased. The obtained difference between Experiments 1 and 2 suggests that a change in item duration subsequent to a buildup of PI in short-term memory does not act in a manner analogous to change in class of material. The effect of a shift in duration is not symmetrical. This finding was repeated in Experiment 3.

One interpretation of the disparity between increasing and decreasing item duration is that the subject modifies his encoding strategy as a function of direction of duration change. It is possible that in the four trials prior to the critical test, the subject developed an encoding strategy whereby he could effectively encode the consonant trigram im the $1.5 \mathrm{sec}$ allotted to him. When the fifth trigram was presented, the subject completed his encoding of this trigram in $1.5 \mathrm{sec}$, then usefully employed the remaining $1 \mathrm{sec}$ to rehearse the trigram. This rehearsal possibility would not have been available to the subject in the decreasing conditions if it is assumed that he developed a strategy of using the full 2.5 -sec duration to read off, once only, the three consonants. Therefore, the duration of the trigram on Trial 5 would be less than the time required by the subject to encode the three consonants.

A second interpretation is also possible. In the present experiments, the presentation of the trigram on Trial 5 in the experimental conditions may be described as novel in the sense that its duration aspect differed from that of the preceding items. Berlyne (1960) argues that a correlate of novelty is arousal. Several experiments suggest that items that are higharousal items (Maltzman, Kantor, \& Langdon, 1966) or items contiguous with arousal states (Berlyne, Borsa, Craw, Gelman, \& Mandell, 1965), are retained better in STM tasks.

In the present series of experiments, novelty on Trial 5 was common to all experimental groups, yet augmented recall occurred only in the increasing duration groups. However, a novelty-arousal interpretation of these data is possible in the framework of the model presented by Sokolov (1960) to account for habituation and the orienting response. Sokolov (1960) proposes that incoming stimuli leave traces of all their 
characteristics, including duration, in the nervous system. These trace configurations are referred to as neuronal models. When a stimulus input does not match the established neuronal models, the orienting response occurs with an overall increase in the sensitivity of receptor mechanisms.

In the case of increasing duration, the neuronal model established over the initial four trials carries information about the linguistic character, visual character, intensity, and duration of the trigram. On Trial 5 there is disparity between the established neuronal model and the presented information, in that the duration of the item is greater than that previously experienced. This disparity can only be detected when the input on Trial 5 extends beyond the previously experienced 1.5 -sec duration. At this point the orienting response is induced and facilitates the reception and encoding of the presented consonant trigram (cf. Maltzman et al., 1966) over the remaining $1 \mathrm{sec}$. However, in the decreasing duration groups, the established neuronal model contains a trace representing a $2.5-\mathrm{sec}$ duration. On presentation of the 1.5-sec trigram on Trial 5, the disparity between the new input and the neuronal model is recognized at the point at which the new input terminates. Therefore, the induced orienting response occurs after the consonant trigram presentation has ended and thus cannot facilitate the reception of this consonant trigram. Berlyne, Borsa, Hamacher, and Koenig (1966) have shown that arousal induced contiguously with the input is superior to arousal induced after the input in terms of the resulting recall level.

In conclusion, it should be noted that the two interpretations suggested above do not claim that temporal extent is necessarily a feature or attribute of items in STM. Rather, the first interpretation suggests that release may be due to modification of encoding strategy, while the second interpretation suggests that arousal may be a determinant of PI release.

\section{REFERENCES}

Berlyne, D. E. Conflict, arousal, and curiosity. New York: McGraw-Hill, 1960.

Berlyne, D. E., Borsa, D. M., Craw, M. A., Gelman, R. S., \& MANDEll, E. E. Effects of stimulus complexity and induced arousal on paired-associate learning. Journal of Verbal Learning and Verbal Behavior, 1965, 4, 291-299.

Berlyne, D. E., Borsa, D. M., Hamacher, J. H., \& Koenig, I. D. Paired associate learning and the timing of arousal. Journal of Experimental Psychology, 1966, 72, 1-6.

Gardiner, J. M., Ulee, H., Redman, G., \& Ball, M. The role of stimulus material in determining release from proactive inhibition. Quarterly Journal of Experimental Psychology, 1976, 28, 395-402.

Maltzman, I., Kantor, W., \& Langdon, B. Immediate and delayed retention, arousal and the orientating and defensive reflexes. Psychonomic Science, 1966, 6, 445-446.

Sokolov, E. N. Neuronal models and the orienting reflex. In M. A. Brazier (Ed.), The central nervous system and behavior. New York: J. Macy, 1960.

WiCKens, D. D. Encoding categories of words: An empirical approach to meaning. Psychological Review, 1970, 77, 1-15.

WICKENS, D. D. Characteristics of word encoding. In A. W. Melton and E. Martin (Eds.), Coding processes in human memory. Washington, D. C: Winston \& Sons, 1972.

Wickens, D. D. Some characteristics of word encoding. Memory \& Cognition, 1973, 1, 485-490.

WRIGHT, J. A. Effects of formal interitem similarity and length of retention interval on proactive inhibition of short-term memory. Journal of Experimental Psychology, 1967, 75, 386-395.

(Received for publication December 19, 1977.) 